\section{IDDF2019-ABS-0150 THERAPEUTIC EFFECT OF FULLY COVERED SELF-EXPANDABLE METAL STENTS ON BENIGN BILIARY STRICTURE}

Like Bie*. Digestive Endoscopy Center, Ruijin Hospital, Shanghai Jiaotong University School of Medicine, China

\subsection{6/gutjnl-2019-IDDFabstracts.95}

Background To evaluate the efficacy and safety of fully covered self-expandable metal stents (FCSEMSs) implanted by endoscopic retrograde cholangiopancreatography (ERCP) for patients with biliary stricture (BBS).

Methods The clinical data of 38 patients with BBS underwent ERCP and FCSEMSs placement between January 2012 and January 2016 was retrospectively recorded. The success rate of BBS, adverse events related to ERCP and stricture recurrence were analyzed.

Results A total of 38 patients underwent FCSEMSs placement with a mean time of stent indwell 8 months (3-13 months). During follow-up after the stent was removed, 1 patient after liver transplantation death was the unrelated cause. The mean follow-up time was 13 months (2-52 months) for other patients. Stricture recurrence occurred in 6 patients, including 2 cases of liver transplantation-related biliary strictures, 1 case of chronic pancreatitis-related biliary stricture. Stricture resolution occurred in 31 cases with $83.8 \%$ (31/37) success rate, including $90.0 \% \quad(18 / 20)$ gallstone-related biliary stricture, $75.0 \% \quad(3 / 4)$ chronic pancreatitis-related biliary stricture, $80.0 \%(8 / 10)$ liver transplantation-related biliary stricture, and $66.7 \%(2 / 3)$ post surgical-related biliary stricture. The incidence of post-ERCP complications was $21.1 \%(8 / 38)$, including mild pancreatitis in 5 cases, hyperamylasemia in 2 cases, cholangitis in 1 case. All patients were cured by conservative treatment.

Conclusions FCSEMSs are effective and safe for BBS. Further studies are needed to assess the indwelling time of FCSEMSs, whether FCSEMSs are superior to multiple plastic stents and the different efficacy of FCSEMSs for BBS caused by different etiology.

\section{IDDF2019-ABS-0158 FECAL MICROBIOTA TRANSPLANTATION PROTECTS LIVER FROM HBV INFECTION}

Jingtong Wu*, Zhangran Chen, Yurou Xie, Xiang Zhang. Institute of Microbial Ecology, School of Medicine, Xiamen University, China

\subsection{6/gutjnl-2019-IDDFabstracts.96}

Background Hepatitis B Virus (HBV) is one of the most common epidemic viruses worldwide. The serological antigen transfusion of chronic HBV infection is still an urgent problem to be solved. Previous results had shown that fecal microbiota transplantation (FMT) has great potential in the treatment of refractory HBV. Immune clearance of $\mathrm{HBV}$ is age-related in both human and mice models. Therefore, we aimed to explore the relationship between the microbiota and HBV chronic tendency.

Methods Adult (12 weeks old) and young (6 weeks old) $\mathrm{C} 3 \mathrm{H}$ mice were hydrodynamically injected with $10 \mu \mathrm{g}$ pAAV/ HBV1.2 plasmid via the tail vein. We gavaged young mice of FMT group three times with Feces from the adult mouse before the tail injection as FMT group, while young mice gavaged with the sterile water served as control (each group,
$\mathrm{N}=5$ ). The serum HBs-Ag level was monitoring weekly after the injection.

Results Rapid HBs-Ag clearance occurred in the adult mice while the control young mice still remained chronic infections. Interestingly, young mice gavaged stool from adult mice showed a large decrease in serum HBs-Ag $(p<0.05)$ in the second week compared to the control young mouse, indicating that FMT may contribute to accelerate the clearance of HBV. In the next 5 weeks, the overall difference of serum HBs-Ag persisted between the two groups of young mice, while the serum HBs-Ag did not show a trend of continuous decline in the FMT mouse.

Conclusions FMT can enhance the capability of the liver to resist the chronic progression of HBV infection. Further research on this phenomenon and its underlying mechanisms may pave a new path for the treatment of HBV.

\section{IDDF2019-ABS-0159 OLIGOFRUCTOSE AMELIORATES NONALCOHOLIC FATTY LIVER DISEASE BY REGULATING GUT MICROBIOTA DYSBIOSIS IN MICE}

Xiaoqing Huang*, Changsheng Yan, Jingtong Wu, Xiang Zhang, Jianlin Ren. Institute of Microbial Ecology, School of Medicine, Xiamen University, China

\subsection{6/gutjnl-2019-IDDFabstracts.97}

Background Intestinal microflora exerts a crucial role in regulating fat metabolism and inflammation. Oligofructose is a water-soluble dietary fiber that cannot be directly digested and absorbed by the human body. It participates in regulating lipid metabolism and alleviating nonalcoholic fatty liver disease (NAFLD)-induced inflammation. However, the underlying mechanisms of oligofructose and intestinal microflora in regulating the progression of NAFLD remain unclear.

Methods A total of $30 \mathrm{C} 57 \mathrm{BL} / 6$ mice were randomly divided into normal diet group (ND group), high-fat diet group (HFD group) and HFD+Oligofructose group (HFD+Oligo group), with 10 mice in each group. After 16-week feeding, blood samples from eyeballs, liver tissues and feces of mice were harvested. Levels of fasting plasma glucose and HOMA-IR of mice were examined. Serum levels of ALT and AST were detected for evaluating the liver damage. H\&E and oil red staining were conducted to assess the level of hepatic steatosis and degree of injury. Distribution and classification of intestinal microflora in mice feces were examined through $16 \mathrm{~s}$ rDNA test.

Results Fasting Plasma Glucose, ALT and AST levels were markedly lower in mice of HFD group relative to those in HFD+Oligo group. H\&E staining revealed that liver inflammation and hepatic steatosis were alleviated in mice of HFD +Oligo group compared with those of HFD group. The distribution of intestinal microflora in mice of HFD+Oligo group was much similar to that of ND group, and the microflora abundances were higher relative to those of HFD group. The distribution of intestinal microflora in mice of HFD +Oligo group was much similar to that of ND group, and the microflora abundances were higher relative to those of HFD group.

Conclusions Oligofructose could regulate intestinal microflora to alleviate the progression of NAFLD. 